\title{
Harnessing the potential of the Kinect sensor for psychiatric rehabilitation for stroke survivors
}

\author{
Melvyn W.B. Zhang ${ }^{1, *}$ and Roger C.M. Ho ${ }^{1}$ \\ Biomedical Institue of Global Healthcare Research and Technology (BIGHEART), National University \\ of Singapore, Singapore
}

Received 7 January 2016

\begin{abstract}
Dominques et al. in their recent article described how low-cost sensors, such as Microsoft Kinect could be utilized for the measurement of various anthropometric measures. With the recent advances in sensors and sensor based technology, along with the rapid advancement in E-health, Microsoft Kinect has been increasingly recognized by researchers and bioengineers to be a low-cost sensor that could help in the collation of various measurements and various data. A recent systematic review done by Da Gama et al. (2015) have looked into the potential of Kinect in terms of motor rehabilitation. The systematic review highlighted the tremendous potential of the sensors and has clearly stated that there is a need for further studies evaluating its potential for rehabilitation. Zhang et al. (2015) in their recent article have advocated several reasons as to why biosensors are pertinent for stroke rehabilitation. Of note, recent studies done by the World Health Organization have highlighted that stroke is a growing epidemic. Aside to the utilization of smartphone based sensors for stroke rehabilitation, as proposed by Zhang et al. (2015), researchers have also investigated the use of other low cost alternatives, such as Kinect, to facilitate the rehabilitation of stroke survivors. Whilst it may seemed like that has been quite extensive evaluation of the Kinect sensor for stroke rehabilitation, one core area that bio-engineers and researchers have not looked into is that of the psychiatric and mental health issues that might at times arise following a stroke. It is thus the aim of this letter to address how such a sensor could be tapped upon for psychiatric rehabilitation amongst stroke survivors. To this end, the authors have thus conceptualized a game that could help in the cognitive remediation for stroke survivors using low cost Kinect sensors.
\end{abstract}

Keywords: Microsoft Kinect, sensors, stroke, stroke survivors, psychiatry, mental health, rehabilitation

Dominques et al. [1] in their recent article described how low-cost sensors, such as Microsoft Kinect, could be utilized for the measurement of various anthropometric measures. The authors proposed a methodology by which the sensor could be utilized for such measurement and highlighted that the information derived from the sensors are comparable to the ones derived from conventional measurement systems [1]. With the recent advances in sensors and sensor based technology, along with the rapid advancement in E-health, Microsoft Kinect has been increasingly recognized by researchers and bioengineers to be a low-cost sensor that could help in the collation of various measurements and various

\footnotetext{
${ }^{1}$ These authors contributed equally.

* Corresponding author: Melvyn Zhang, Biomedical Institue of Global Healthcare Research and Technology (BIGHEART), National University of Singapore, 119074, Singapore. Tel.: +65 63892504; Fax: +65 63892222; E-mail: melvynzhangweibin @gmail.com.
} 


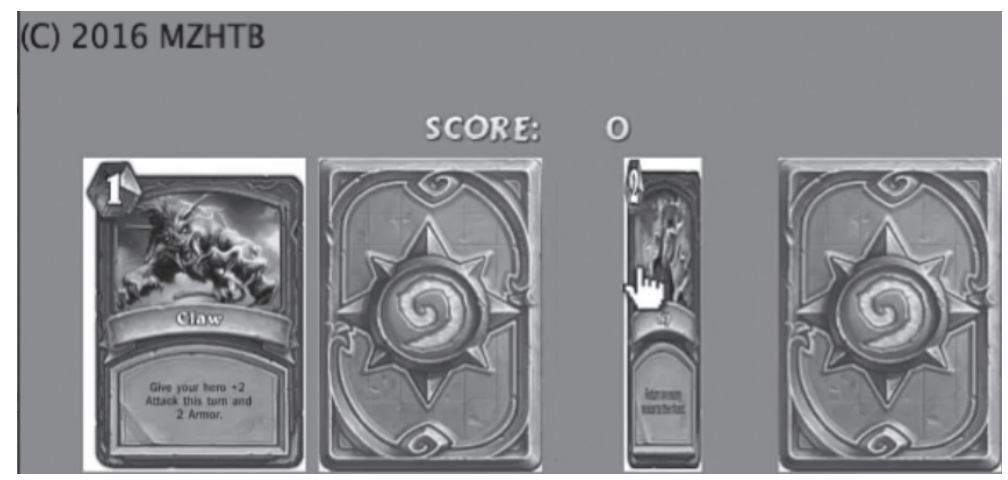

Fig. 1. Overview of card memory game.
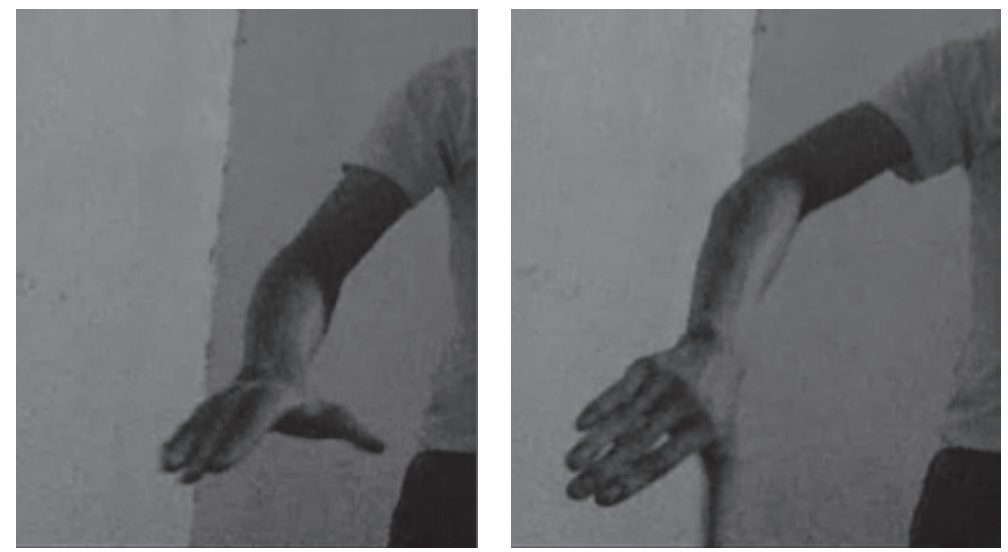

Fig. 2. Overview of how the game could be controlled using postural movements.

data. This sensor has been recognized as being of use for the accurate determination of body motion [2], and this would have a consequential utilization for sport analysis [2]. The same sensor has been proposed to be also capable of being used for head pose estimation and might help in bio-systems involving facial recognition as well as yawn detection [3]. A recent systematic review done by Da Gama et al. [4] have looked into the potential of Kinect in terms of motor rehabilitation. Based on the previously published systematic review, which looked at a cumulative total of 31 articles, there were a total of at least 13 articles that focused on the development and implementation of the sensor for rehabilitation. The systematic review highlighted the tremendous potential of the sensors and has clearly stated that there is a need for further studies evaluating its potential for rehabilitation.

Zhang et al. [5], in their recent article have advocated how sensors in the smartphone could potentially be tapped onto and be used for Stroke rehabilitation, instead of the need for there to have new biosensors, which will be costly for patients to own. Zhang et al. [5] in their article have advocated several reasons as to why biosensors are pertinent for stroke rehabilitation. Of note, recent studies done by the World Health Organization have highlighted that stroke is a growing epidemic [6]. In addition, stroke survivors tend to face much disability as the condition in itself would affect their general mental health well being due to functional losses as well as their quality of life, as they now are dependent on others to clear for them. Aside to the utilization of smartphone based sensors for stroke rehabilitation, as proposed by Zhang et al. [5], researchers have also investigated the use of other low cost alternatives to facilitate the 
rehabilitation of stroke survivors. Mintal et al. [7] have described how they have managed to make use of C\# language as well as WPF technology in the creation of an application for movement therapy. Mobini et al. [8] have described how they have tapped onto the Kinect sensor for the accurate evaluation of the upper body recovery of stroke patients. Whilst it may seemed like that has been quite extensive evaluation of the Kinect sensor for stroke rehabilitation, one core area that bio-engineers and researchers have not looked into is that of the psychiatric and mental health issues that might at times arise following a stroke. Oliveria et al. [9] in their recent article highlighted the association between a right hemispheric stroke and the resultant cognitive and memory deficits. It is believed that those with a right hemispheric stroke and signs of depression do have impaired performance across a multitude of cognitive domains. Other prior studies have highlighted that as many as $74 \%$ of individuals with cortical stroke and as many as $46 \%$ of individuals with subcortical stroke suffer from cognitive and memory deficits [10]. As the resultant cognitive impairment has a impact on the functional recovery after a stroke [10], cognitive training has been recommended for most stroke survivors. With the advances in technology, to date, this could be done using various software programs [10]. The core exercises that are routinely done in cognitive training include that of time orientation, spatial orientation, visual attention, logical reasoning, memory and executive functioning exercises.

As discussed previously, much of the work involving the low cost sensor (Kinect) has been largely focused on physical and motor rehabilitation. To this end, the authors have thus conceptualized a game that could help in the cognitive remediation for stroke survivors using low cost sensors. The cost of a Kinect sensor is estimated to be around USD 160 dollars. The game that the authors have conceptualized is made available on the Windows platform. Most of the current intervention that have been developed would still require the user to purchase the gaming console, which would add on to the cost of the biosensor in itself. In our conceptualization, all the stroke survivor has to do is to purchase the Kinect sensor and get it connected to their existing computer. Instead of relying on the SDK (Software Development Kit) provided by Microsoft for developers, the authors have made of a cross-platform gaming development kit, Unity 3D [11]. By doing so, the game that is developed could also run on other operating systems as well. The memory game that is developed seeks to address various cognitive issues, in particular that of spatial orientation, as well as memory and executive functioning. In addition, through the usage of the sensor, while the stroke survivor is doing tasks to help in cognitive remediation, he is to some extent also engaging in tasks that facilitate physical rehabilitation. In our conceptualization, the stroke survivor would need to move his or her thumb to move the respective cards. By doing so, the authors hope that this would enable them to be re-trained with regards to spatial orientation, as well as enable them to recognize and remember the positions of the cards. The stroke survivor would score a point only if they are able to match the right cards together. To some extent, stroke survivors would have to rely on visual memory and attention as well, as they need to avoid the different cards, which are meant to be distractors.

In conclusion, the Kinect sensor has been extensively evaluated in current literature, which support its usage in physical rehabilitation and the assessment of gait and postures. While the smartphone sensors could be tapped upon as well for stroke physical rehabilitation, it is of importance for researchers to recognize that the Kinect sensor could also be particularly useful for stroke survivors in terms of rehabilitation. Most studies have focused largely on the usage of Kinect sensors for physical rehabilitation, but it is essential to note that stroke survivors do also have associated psychiatric comorbidities. It is thus the aim of this letter to address how such a sensor could be tapped upon for psychiatric rehabilitation amongst stroke survivors. 


\section{Conflict of interest}

The authors declare that they have no competing interests.

\section{References}

[1] Domingues A, Barbosa F, Pereira EM, Santos MB, Seixas A, Vilas-Boas J, Gabriel J, Vardasca R. Towards a detailed anthropometric body characterization using the Microsoft Kinect. Technol Health Care. 2015 Nov 17. [Epub ahead of print]

[2] Gao Z, Yu Y, Zhou Y, Du S. Leveraging Two Kinect Sensors for Accurate Full-Body Motion Capture. Sensors (Basel). 2015 Sep 22; 15(9): 24297-317. doi: 10.3390/s150924297.

[3] Saeed A, Al-Hamadi A, Ghoneim A. Head Pose Estimation on Top of Haar-Like Face Detection: A Study Using the Kinect Sensor. Sensors (Basel). 2015 Aug 26; 15(9): 20945-66. doi: 10.3390/s150920945.

[4] Da Gama A, Fallavollita P, Teichrieb V, Navab N. Motor Rehabilitation Using Kinect: A Systematic Review. Games Health J. 2015 Apr; 4(2): 123-35. doi: 10.1089/g4h.2014.0047. Epub 2015 Feb 6.

[5] Zhang MW, Chew PY, Yeo LL, Ho RC. The untapped potential of smartphone sensors for stroke rehabilitation and after-care. Technol Health Care. 2015 Oct 12. [Epub ahead of print]

[6] Thomas T, Stephen B, Colin M. The global burden of cerebrovascular disease. Extracted from www.who.int/healthinfo/ statistics/bod_cerebrovasculardiseasestroke.pdf Last assessed on 12th September 2015.

[7] Mintal FA, Szucs V, Sik-Lanyi C. Developing movement therapy application with Microsoft Kinect control for supporting stroke rehabilitation. Stud Health Technol Inform. 2015; 217: 773-81.

[8] Mobini A, Behzadipour S, Saadat M. Test-retest reliability of Kinect's measurements for the evaluation of upper body recovery of stroke patients. Biomed Eng Online. 2015 Aug 4; 14: 75. doi: 10.1186/s12938-015-0070-0.

[9] Oliveira CR, Pagliarin KC, Calvette Lde F, Gindri G, Argimon II, Fonseca RP. Depressive signs and cognitive performance in patients with a right hemisphere stroke. Codas. 2015 Oct; 27(5): 452-7. doi: 10.1590/2317-1782/20152015005.

[10] Zucchella C, Capone A, Codella V, Vecchione C, Buccino G, Sandrini G, Pierelli F, Bartolo M. Assessing and restoring cognitive functions early after stroke. Funct Neurol. 2014 Oct-Dec; 29(4): 255-62.

[11] Unity 3D. Extracted from: https://unity3d.com, last assessed on 7th January 2016. 